\title{
EVARISTO GALOIS
}

\author{
CARLOS PORTILLA SILVA \\ JAVIER CIFUENTES SUAREZ \\ (alumnos licenciatura)
}

Evaristo Galois nació el 25 de Octubre de 1811 en Bourg-La-Reine. El revuelto ambiente político-social que le correspondió vivir (su padre fué alcalde en sus ciudad natal) parece haber influído fuertemente en su conducta ciudadana. Su alto espíritu crítico y la gran impetuosidad de su juventud, le significaron grandes problemas, incluso le costaron la vida.

En 1832 entró a estudiar en el Lycée Louis- leGrand en Paris. A pesar de su luminoso genio matemático, fué claramente un estudiante difícil. Reprobó dos veces los exámenes de admisión a la Ecole Polytechnique y fué aceptado en la Ecole Normal en 1830. No alcanzó a permanecer un año en ese lugar de estudios pues fué expulsado por causa de una publicación en la cual criticaba las acciones en que se vió envuelto el director durante la Revolución de Julio. 
Al año siguiente (con 19 años de edad) fué arrestado por tratar de hablar en contra del Rey Louis Philippe, pero fué perdonado. Poco tiempo después recibió una sentencia a seis meses de prisión por uso ilegal de uniforme y por portar armas. En 1832, el 31 de Mayo, cuando sólo tenía 20 años de edad, muere a causa de un duelo, posiblemente con un agente provocador de la policía.

A pesar de su corta existencia, sus trabajos han entregado una de las teorías más penetrantes en las matemáticas actuales, la Teoría de Galois. Además de algunos cortos papers que publicó, presentó tres trabajos más amplios (acerca de la teoría de ecuaciones) que fueron rechazados por la Academia Francesa. En la víspera de su fatal duelo escribió una carta a su amigo Augusto Chevalier en la cual bosqueja el contenido de tres temas matemáticos. Uno de ellos es acerca de integrales elípticas e integrales de funciones algebraicas. Los otros dos tratan de la teoría de ecuaciones.

Usando el lenguaje actual de la matemática, la teoría de Galois puede resumirse como sigue:

Si $f(x) \varepsilon Q[x]$ es irreducible sobre $Q$, entonces los ceros $\mathrm{x} 1, \mathrm{x} 2, \mathrm{xn}$ son números complejos (o reales) no racionales. Si E es el menor cuerpo que contiene $Q$ y todos los ceros de $f(x)$, es posible probar que $\mathrm{G} Q=\{$ automorfismos de E que dejan fijos elementos de $Q$ \} es un grupo de Galois de $f(x)$, bajo la composición de funciones. Galois probó que existe una correspondencia uno a uno entre los subcuerpos de $E$ que contienen $Q$ y los sub-grupos de $G Q$. Este hecho constituye el teorema fundamental de la teoría de Galois y su importancia en el álgebra va mucho más le- 
jos que su aplicación a la teoría de ecuaciones. Galois también probó que una ecuación algebraica de la forma $f(x)=0$ es soluble por radicales si y solo si e grupo de Galois de $f(x)$ es un grupo soluble. Como es sabido, el grupo Sn no es soluble para $\mathrm{n} \geq 5$, y como para cada $\mathrm{n} \geq 5$ existe alguna ecuación cuyo grupo de Galois corresponde a $\mathrm{Sn}$, se tienen que esas ecuaciones no pueden ser resueltas por radicales.

En consecuencia Galois probó que es imposible encontrar la solución de an $\mathrm{xn}+\ldots+$ ao $=0$, mediante una fórmula en término de los coeficientes, cuando $n \geq 5$. Este resultado ya lo habían demostrado en 1824, en forma independiente Paolo Ruffini (1765-1822) y N.H. Abel (1802 1829). E1 trabajo de Galois es conclusivo porque obtuvo un criterio general para la solubilidad de ecuaciones algebraicas mediante radicales. Lodovico Ferrari (15221562) resolvió la ecuación de cuarto grado y Niccoló Tastaglia (1499-1559) la de tercer grado aún cuando esta última fué publicada por Girolomo Cardano (1501-1576) en el Ars Magna (1545).

FUENTE BIBLIOGRAFICA: Enciclopedia Británica (1968) COLLIER'S Encyclopedia (1966)

LAROUSSE 